\title{
Resonance Absorption of Light By Subwavelength Diffractive Gratings
}

\section{N.l. Petrov¹, V.A. Danilov¹, V.V. Popov², and B.A. Usievich ${ }^{3}$}

${ }^{1}$ Scientific and Technological Center of Unique Instrumentation of Russian Academy of Sciences, Butlerova str., 15, Moscow 117342, Russia

${ }^{2}$ Moscow State University, Moscow, Russia

${ }^{3}$ General Physics Institute of RAS, Moscow, Russia

\section{Abstract}

Diffraction of light of a visible spectral range by subwavelength metal gratings is investigated theoretically and experimentally. The influence of different grating parameters (filling factor, shape and depth, material, angle of incidence, wavelength and polarization of radiation) on diffraction efficiency is investigated. The conditions are found under which there are only zero diffraction order and the minus first order.

Corresponding Author:

N.I. Petrov

petrovni@mail.ru

Received: 28 January 2018

Accepted: 15 March 2018

Published: 25 April 2018

Publishing services provided by Knowledge $\mathrm{E}$

(c) N.I. Petrov et al. This article is distributed under the terms of the Creative Commons

Attribution License, which permits unrestricted use and redistribution provided that the original author and source are credited.

Selection and Peer-review under the responsibility of the PhIO Conference Committee. It is established that the zero order can be suppressed by selecting the depth and shape of the grating relief. High diffraction efficiency in the -1st order is observed with increasing depth of the grating relief (more than $70 \%$ at a depth $h=80 \mathrm{~nm}$ ). It is shown that under certain conditions an effect of plasmon resonance occurs, at which there is a complete absorption of the incident radiation. The considered optical elements can be used in systems for image processing, projection displays, in the development of a variety of sensors, etc.

Keywords: subwavelength grating, diffraction efficiency, plasmon resonance

\section{Introduction}

The interest in subwavelength gratings is growing due to their promising applications in high performance transmission and reflection filters [1], optoelectronic devices using surface plasmons [2-4], spectral-selective external optical mirrors for lasers (VCSELs) [5]. Subwavelength gratings have also been proposed for focusing light in the far [6], and near zones [7].

Subwavelength dielectric gratings are an alternative to distributed Bragg multilayer dielectric reflectors for broadband highly reflecting applications at the radiation filtration. These structures are compact and cheap to manufacture and provide new opportunities for control of polarization properties of radiation. The diffraction gratings 
also find application in devices for splitting the light beam into colour components [814]. In [15] a new type of thin film device to separate colour beams based on the effect of frustrated total internal reflection was considered. Such optical elements can be used for image processing in imaging systems, projection displays, etc.

In [16] subwavelength gratings were proposed and demonstrated for combining red, green and blue light beams into a single beam. It was shown that such gratings have a high efficiency and can significantly reduce the size of the projection devices used in mobile phones, etc. The efficiency of such devices is affected by several factors that depend on material properties and structural parameters of the grating such as period, fill factor, and depth of the microrelief.

Multilayer dielectric distributed Bragg reflectors are commonly used for highly reflecting broadband applications in filtering radiation. The metal-dielectric plasmonic structures for frequency-selective and broadband absorbers are also of practical interest. Such structures for the complete absorption of radiation in the far IR region were considered in [17].

In the present work it is shown that the effect of full absorption can be also observed in sub-wavelength metallic gratings for radiation in the visible range of the spectrum. The diffraction efficiencies of the gratings made of various metals ( $\mathrm{Ni}, \mathrm{Ag}, \mathrm{Al}$, etc.) with different depths of the profile are calculated and measurements are carried out. The good agreement of measurement results with calculations is obtained. It is shown that under certain conditions there is an effect of plasmon resonance. Subwavelength gratings with a period of $400 \mathrm{~nm}$ and $600 \mathrm{~nm}$ are fabricated and measurements using lasers and laser diodes that emit in the visible wavelength range $(450-650 \mathrm{~nm}$ ) are conducted.

\section{Simulation results}

Since the scalar diffraction theory cannot be used in the case of subwavelength gratings [18], the electromagnetic theory [19] based on C-method [20, 21] and the method of rigorous analysis of coupled waves (RCWA method) [22] are used for the calculations.

RCWA method (rigorous coupled wave approach) is based on the solution of the Maxwell equations in a truncated Fourier space. Because of the periodicity, each component of the tensor of the dielectric permittivity can be expanded in a Fourier series. 
Wave propagation in periodic structures is described by the wave Helmholtz equation

$$
\Delta E(x, z)+k_{0}^{2} \epsilon(x) E(x, z)=0,
$$

where $\varepsilon(x)$ is the periodic function of the dielectric constant, which can be represented in the form of an infinite series

$$
\epsilon(x)=\sum_{n=-\infty}^{\infty} \gamma_{n} \exp (\operatorname{inh} x)
$$

Here $h=2 \pi / d$ is the minimum value of the vector of the reciprocal lattice, which is directed along the $x$-axis and the $z$ coordinate is directed perpendicularly to the surface of the grating.

The coefficients $\gamma_{n}$ in (2) are determined by the Fourier integral within the grating period $d$.

It is convenient to place the origin of the coordinate system in the middle of the layer with the thickness $d$ :

$$
\gamma_{n}=\frac{1}{d} \int_{-d / 2}^{d / 2} \epsilon(x) \exp (-i n h x) d x
$$

The field inside the periodic medium is represented as a superposition of plane waves. The method of calculation consists of two stages. The first stage involves finding eigenwaves of each layer within the medium. Maxwell's equations for each layer are recorded in the Fourier space taking into account the decomposition of the tensor of the dielectric permittivity in a Fourier series and decomposition of the electric and magnetic fields components on plane-waves. In the practical implementation a truncated Fourier space is used, the dimension of which is determined by the condition of convergence of the obtained results. In the second stage after crosslinking the solutions in the boundary layers, we obtain the system of linear algebraic equations for finding the components of the reflected and transmitted electromagnetic field in all diffraction orders.

\subsection{Diffraction efficiency}

The diffraction efficiency of the grating is affected by a number of conditions: fill factor, shape and depth, material, angle of incidence, wavelength and polarization of radiation.

In Fig. 1 the diffraction efficiencies in the dependence on wavelength are presented for various materials. The angle of incidence of the radiation with TM polarization on a 
grating is of 60 degrees. The grating in the form of an isosceles triangle is considered. The altitude of the triangle (depth of the microrelief) is equal to $160 \mathrm{~nm}$. The grating period is $\Lambda=400 \mathrm{~nm}$. Materials - aluminium, chromium, silver, gold, copper, molybdenum. As follows from the calculations, the change of the diffraction efficiency is stronger for blue colour. It is seen that the highest diffraction efficiencies are achieved with the use of gratings made of silver and aluminum.
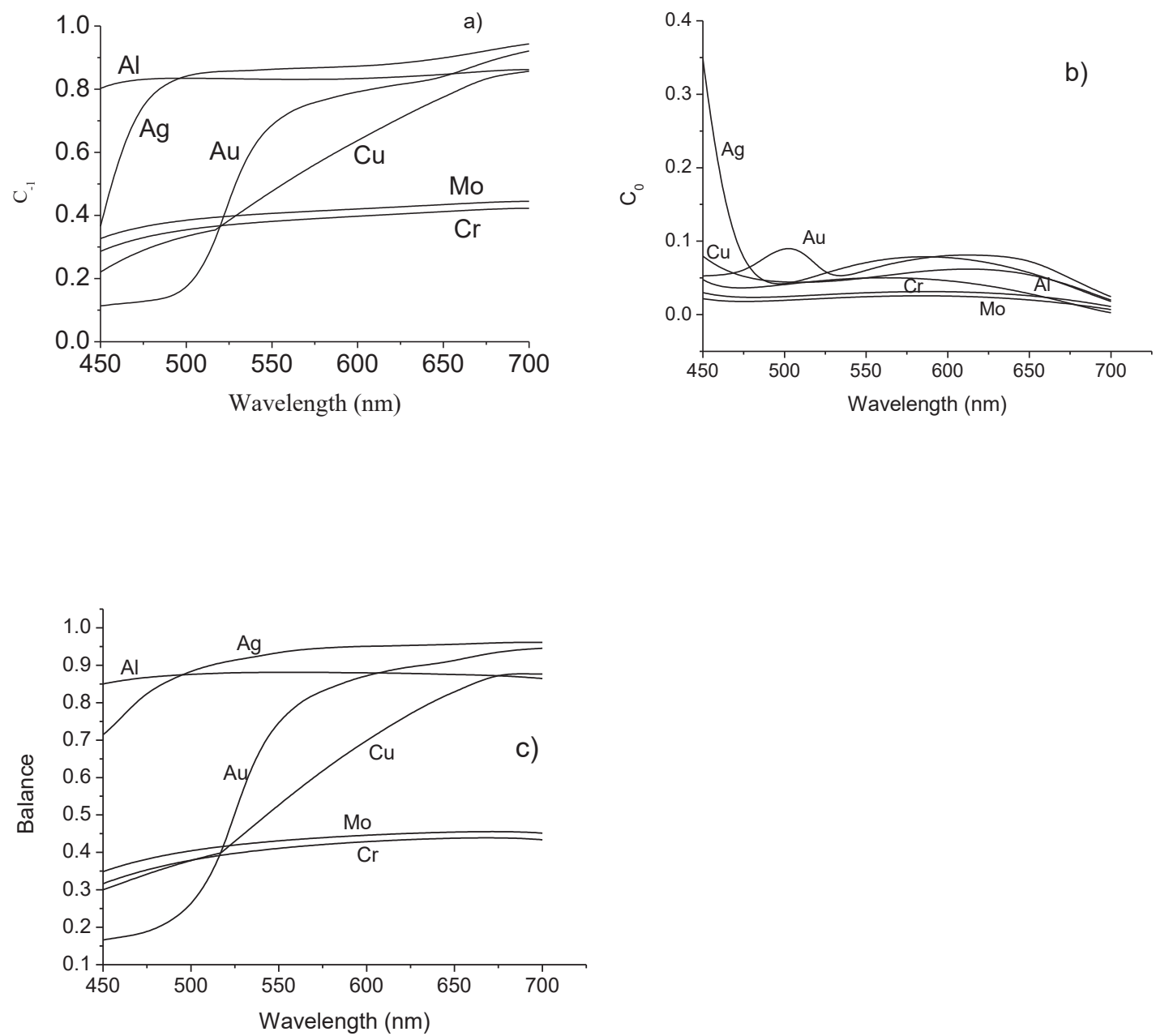

Figure 1: Diffraction efficiencies at minus first diffracted order $C_{-1}(a)$, zero reflected order $C_{0}(b)$ and total intensity $C_{-1}+C_{0}(c)$ depending on the wavelength of the radiation for various materials.

The angles of incidence of light beams can be determined from the equation for gratings:

$$
\sin \theta_{i}+\sin \theta_{w}=\frac{m \lambda_{i}}{\Lambda}
$$

where $\theta_{i}$ is the incidence angle for red, green and blue light beams; $\theta_{w}$ the angle of reflection of the combined light beam; $\lambda_{i}$ is the wavelength of red, green and blue light beams, respectively; $m= \pm 1, \pm 2, \pm 3, \ldots$ is the diffraction order; $\Lambda$ is the grating period. 
For the angle of incidence of the light beam we obtain the following expression from (4):

$$
\theta_{i}=\arcsin \left(\frac{m \lambda_{i}}{\Lambda}-\sin \theta_{w}\right)
$$

\subsection{Surface plasmons}

The light excites surface plasmons through a grating [23]. The excitation of surface plasmons upon noncollinear scattering on a metallic diffraction grating was studied in [3]. In [4], it is shown that the resonance features in the spectral-angular dependencies of transmittance of nano-gratings are due to the excitation of surface plasmons at metal-air and metal-dielectric boundaries.

If the grating constant is equal to $\Lambda$, then the wave vector of the light acquires an additive $2 \pi / \Lambda$, and the dispersion relation for surface plasmons should take into account the component of the wave vector which is parallel to the surface. For the angle of incidence $\theta_{0}$, the resonance condition takes the form:

$$
\frac{\omega}{A} \sin \theta_{0}+\frac{2 m \pi}{\Lambda}=\frac{\omega}{c} \sqrt{\frac{\epsilon_{r}}{\epsilon_{r}+1}},
$$

where $\epsilon_{r}$ is the real part of the dielectric constant of the grating material, $m$ is the integer.

Note that this equation does not contain the depth of the grating, and for deep gratings it can be considered only as the estimation equation. However, the values of the resonance angles obtained from this equation are in good agreement with the exact calculations. Using (6), it is possible to determine the values of the angles of incidence of the beam on the grating at which the plasmon resonance occurs. Table 1 shows the values of the angles of incidence of the red, green and blue beams for $A l$, $\mathrm{Ni}$ and $\mathrm{Ag}$ gratings.

TABLE 1: The angles of incidence satisfying the condition for the appearance of the plasmon resonance ( $\Lambda$ $=400 \mathrm{~nm}$ ) for various metals.

\begin{tabular}{|c|c|c|c|} 
& $\lambda=641 \mathrm{~nm}$ & $\lambda=532 \mathrm{~nm}$ & $\lambda=450 \mathrm{~nm}$ \\
\hline$\theta_{0}(\mathrm{Al})$ & $36.4^{\circ}$ & $18.5^{\circ}$ & $6.2^{\circ}$ \\
\hline$\theta_{0}(\mathrm{Ni})$ & $33.4^{\circ}$ & $14.5^{\circ}$ & $0.57^{\circ}$ \\
\hline$\theta_{0}(\mathrm{Ag})$ & $34.8^{\circ}$ & $16.1^{\circ}$ & $1.9^{\circ}$ \\
\hline
\end{tabular}

In Fig. 2 the diffraction efficiencies in various orders of nickel $(a, b)$ and silver $(c, d)$ sinusoidal gratings with periods $\Lambda=600 \mathrm{~nm}$ and $\Lambda=400 \mathrm{~nm}$ depending on the angle of 
incidence of radiation of TM polarization with a wavelength of $\lambda=641 \mathrm{~nm}$ at different values of the depth of the microrelief are presented. At a certain angle of incidence there is the effect of plasmon resonance. It is seen that when the relief depth of $h=80$ $\mathrm{nm}$ and angle of incidence $\theta_{i}=33^{\circ}$ almost $100 \%$ of the incident energy is absorbed by the grating made of silver (Fig. $2 d$ ). For the nickel grating full absorption of the incident radiation is achieved with a relief depth of $h=50 \mathrm{~nm}$ and angle of incidence $\theta_{i}=31^{\circ}$ (Fig. 2b).
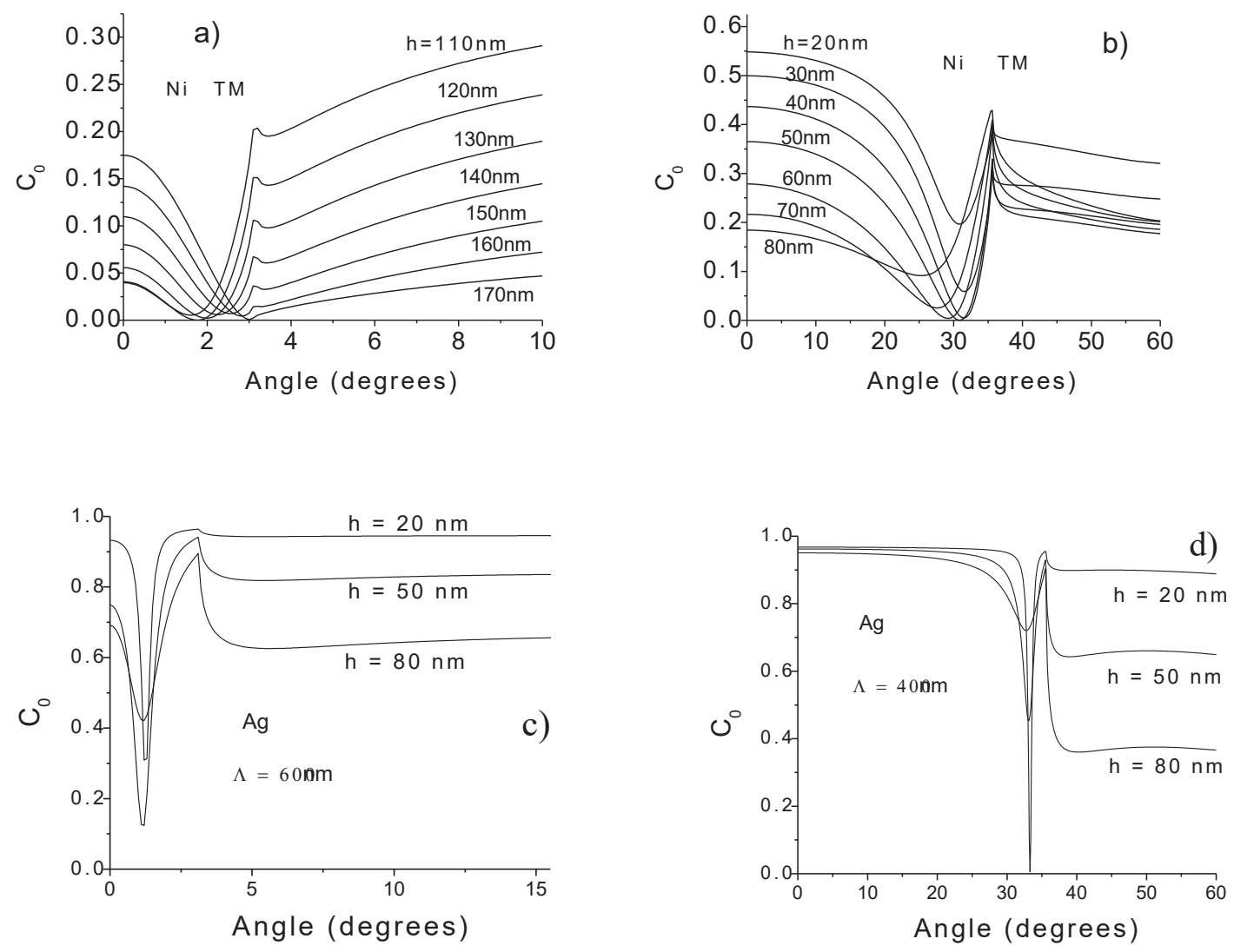

Figure 2: Diffraction efficiencies in the zero order depending on the incidence angle for different depths of microrelief gratings of nickel $(a, b)$ and silver $(c, d)$ at the radiation wavelength of $641 \mathrm{~nm}:(\mathrm{a}, \mathrm{c}) \Lambda=$ $600 \mathrm{~nm} ;(b, d) \Lambda=400 \mathrm{~nm}$.

\section{Measurement Results}

Two groups of samples were investigated: the original gratings and their nickel replicas. The original gratings were fabricated using electron-beam lithography [24] and etching in a film of polymethylmethacrylate (PMMA) deposited on chrome glass substrate using spin-coating technology [25]. In Fig. 3 the image of the grating with a period of $\Lambda=400 \mathrm{~nm}$ obtained by atomic force microscope (AFM) is shown. As follows 
from AFM measurements, the shape of the profile of the grating is well described by the trapezoidal model.

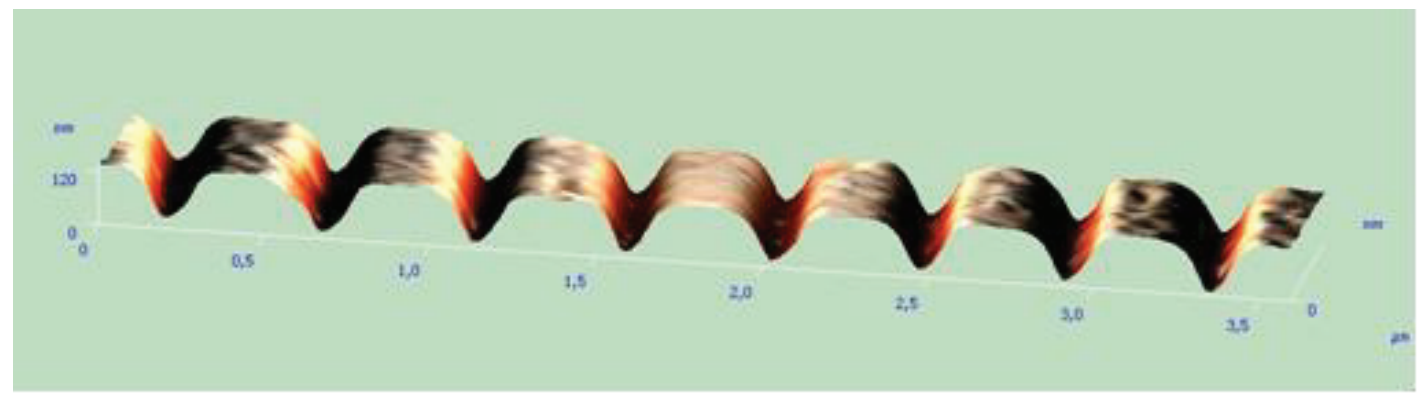

Figure 3: The grating image, obtained with an atomic force microscope (AFM). The period is of $400 \mathrm{~nm}$, a height of $110 \mathrm{~nm}$.

In Fig. 4 the results of calculations and measurements of diffraction efficiency of the zero order of the nickel grating depending on the angle of incidence of the radiation with $p$ - polarization at different values of the depth of the microrelief are presented. It is seen that the effect of plasmon resonance at the incidence angle of $\sim 33^{\circ}$ takes place. With a relief depth of $h=80 \mathrm{~nm}$ and angle of incidence $\theta_{i}=31^{\circ}$ almost all incident energy is absorbed by the grating. Note that the measured angles of incidence, at which the plasmon resonance effect occurs, are in good agreement with the calculations.
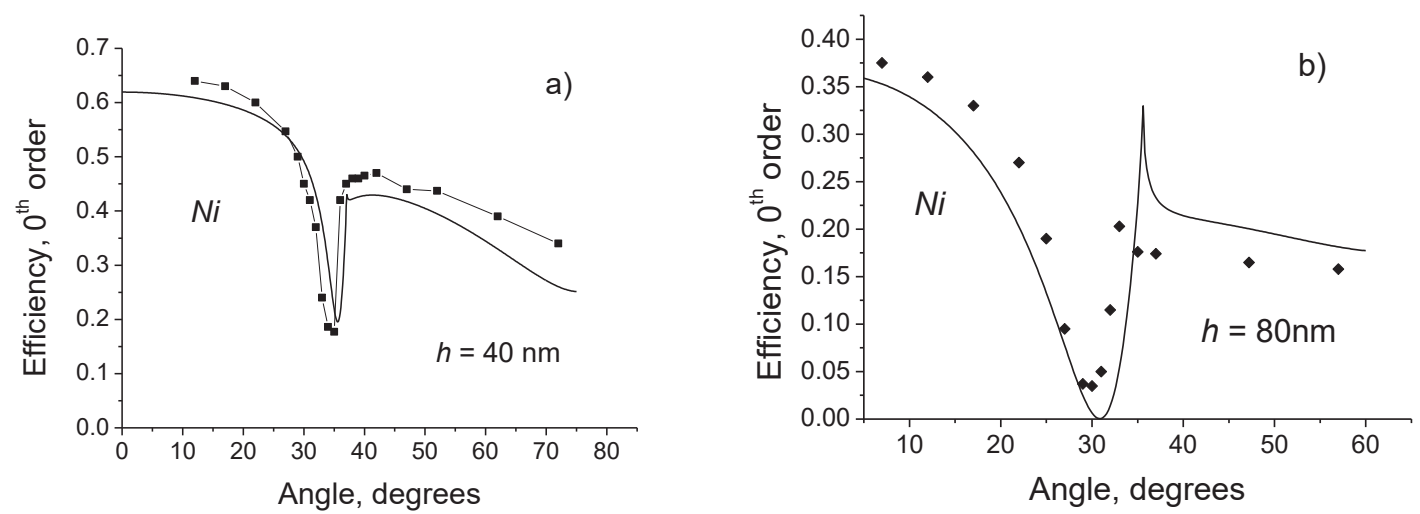

Figure 4: Calculated (solid lines) and measured diffraction efficiencies of the zero order depending on the angle of radiation incidence for the nickel grating with a period of $\Lambda=400 \mathrm{~nm}$ and a depth of $h=40 \mathrm{~nm}$ (a) and $h=80 \mathrm{~nm}(\mathrm{~b})$ at a wavelength of radiation $\lambda=641 \mathrm{~nm}$ with $p$ - polarization.Puc. 1.

\section{Conclusions}

Thus, studies have shown that it is possible to obtain high diffraction efficiency for subwavelength gratings in a wide spectral range $(450-650 \mathrm{~nm})$. The advantage of subwavelength gratings compared with conventional gratings is that there are only 
the zero diffraction order and the minus first order. The zero order can be suppressed by selecting the depth and shape of the relief. This allows high efficiency of minus first diffraction order in a wide spectral range for the same grating to be obtained. Such gratings can find application in many fields such as sensors and pico-projectors, where efficiency and compactness are the determining factors. The obtained results can be useful for development of new devices for combining [26] and/or splitting beams of light, and also devices based on the effect of the excitation of plasmons [27, 28]. Such optical elements can be used for image processing in imaging systems, 3D displays, projection displays, etc.

When the plasmon resonance occurs it is possible to find the optimal grating parameters (material, depth) and radiation (the angle of incidence, polarization), at which the reflected and diffracted light is totally absent, i.e. $100 \%$ absorption of the energy of the incident radiation of the visible range by metallic subwavelength grating takes place. This property can be used to develop various devices, in particular, in solar cells and displays.

\section{Acknowledgement}

The financial support of this research by the Russian Science Foundation (project No. 17-19-01461) is highly appreciated.

\section{References}

[1] S. Tibuleac, R. Magnusson, "Reflection and transmission guided-mode resonance filters," J. Opt. Soc. Am. A, vol. 14, pp. 1617- 1626, 1997.

[2] Ghaemi H.F., Thio T., Grupp D.E., Ebbesen T.W., Lezec H.J., "Surface plasmons enhance optical transmission through subwavelength holes," Phys. Rev. B, vol. 58, pp. 67796782, 1998.

[3] A.V. Andreev, A.A. Korneev, L.S. Mukina, M.M. Nazarov, I.R. Prudnikov, A.P. Shkurinov, "Peculiarities of excitation of surface plasmons upon noncollinear light scattering," Quantum Electronics, vol. 35, pp. 27-32, 2005.

[4] A. V. Andreev, Yu. V. Grishchenko, M. I. Dobynde, T. V. Dolgova, et. al.," Optical properties of one-dimensional subwave plasmonic nanostructures," JETP Letters, vol. 92, pp. 742-745, 2010.

[5] S. Block, E. Gamet, F. Pigeon, "Semiconductor laser with external resonant grating mirror," IEEE J. Quantum Electron., vol. 41, Pp. 1049-1053, 2005. 
[6] D. Fattal, J. Li, Z. Peng, M. Fiorentino, R.G. Beausoleil, "Flat dielectric grating reflectors with focusing abilities," Nature Photonics, vol. 4, pp. 466-470, 2010.

[7] Y.C. Cheng, H. Zeng, J. Trull, C. Cojocaru, M. Malinauskas, T. Jukna, D.S. Wiersma, K. Staliunas, "Beam focalization in reflection from flat dielectric subwavelength gratings," Opt Lett., vol. 39, pp. 6086-6089, 2014.

[8] H. Dammann, "Color separation gratings," Applied Optics, vol. 17, 2273-2279, 1978.

[9] M.W. Farn, M.B. Stern, W.B. Veldkamp, S.S. Medeiros, "Color separation by use of binary optics," Optics Letters, vol. 18, 1214-1216, 1993.

[10] Chun-Wei Liu, Chi-Hung Lee, Tzu-Chun Yang, Chia-Jen Ting, Tsung-Hsin Lin, ShihChieh Lin, "Submicrometer grating light bar for a color-separation backlight," Applied Optics, vol. 52, pp. 3617-3623, 2013.

[11] W.B. Veldkamp, J.R. Leger, G.J. Swanson, "Coherent summation of laser beams using binary phase gratings," Optics Letters, vol. 11, pp. 303-305, 1986.

[12] E.C. Cheung, J.G. Ho, G.D. Goodno, R.R. Rice, J. Rothenberg, P. Thielen, M. Weber, M. Wickham, "Diffractive-optics-based beam combination of a phase-locked fiber laser array," Optics Letters, vol. 33, pp. 354-357, 2008.

[13] T. Shiono, T. Hamamoto, K. Takahara, "High-efficiency blazed diffractive optical elements for the violet wavelength fabricated by electron-beam lithography," Applied Optics, vol. 41, pp. 2390-2393, 2002.

[14] N. Destouches, A.V. Tishchenko, J.C Pommier, S. Reynaud, O. Parriaux, S. Tonchev, M. Abdou Ahmed, "99\% efficiency measured in the -1st order of a resonant grating, “Opt. Express, vol. 13, no. 9, pp. 3230-3235, 2005.

[15] N.I. Petrov, "Frustrated-total-internal-reflection-based thin-film color separator," Optics Letters, vol. 32, pp. 2744-2746, 2007

[16] N.I. Petrov, V.G. Nikitin, V.A. Danilov, V.V. Popov, B.A. Usievich, "Subwavelength diffractive color beam combiner," Applied Optics, vol. 53, pp. 5740-5744, 2014.

[17] B. Vial, G. Demesy, F. Zolla, et.al., "Resonant metamaterial absorbers for infrared spectral filtering: quasimodal analysis, design, fabrication, and characterization," JOSA B, vol. 31. pp. 1339-1346, 2014.

[18] D.A. Gremaux, N.C. Gallagher, "Limits of scalar diffraction theory for conducting gratings," Appl. Opt., vol. 32, pp. 1948-1953, 1993.

[19] N. Lyndin 'MC Grating Software Development Company, 'http://www.mcgrating.com/.

[20] J. Chandezon, D. Maystre, G. Raoult, "A new theoretical method for diffraction gratings and its numerical application," J. Optics (Paris), vol. 11, pp. 235-241, 1980. 
[21] J. Chandezon, M.T. Dupuis, G. Cornet, "Multicoated gratings: a differential formalism applicable in the entire optical region," J. Opt. Soc. Am., vol. 72, pp. 839-846, 1982.

[22] M.G. Moharam, E.B. Grann, D.A. Pommet, "Formulation for stable and efficient implementation of the rigorous coupled-wave analysis of binary gratings," J. Opt. Soc. Am. A, vol. 12, pp. 1068-1076, 1995.

[23] H. Raether. Surface Plasmons on Smooth and Rough Surface and on Gratings (Springer-Verlag, Berlin, 1988).

[24] T. Fujita, H. Nishihara, J. Koyama, "Blazed gratings and Fresnel lenses fabricated by electron-beam lithography," Optics Letters, vol. 7, pp. 578-580, 1982.

[25] T. Novikova, A. De Martino, P. Bulkin, Q. Nguyen, B. Drevillon, V. Popov, A. Chumakov, "Metrology of replicated diffractive optics with Mueller polarimetry in conical diffraction," Optics Express, vol. 15, pp. 2033-2046, 2007.

[26] Weichung Chaoa, Sien Chi, Ching Yi Wu, Chung J. Kuo, "Computer-generated holographic diffuser for color mixing," Optics Communications, vol. 151, pp. 21-24, 1998.

[27] Xiaoliang Sun, Xuewen Shu, and Changhong Chen, "Grating surface plasmon resonance sensor: angular sensitivity, metal oxidization effect of Al-based device in optimal structure," Applied Optics, vol. 54, pp. 1548-1554, 2015.

[28] N.I. Petrov, V.A. Danilov, V.V. Popov, B.A. Usievich, "Excitation of surface plasmons in subwavelength gratings," Frontiers in Optics/Laser Science Conference (FiO/LS), Washington, USA, 2017, Paper JW3A.107. 